\title{
Gobernanza con análisis territorial en Emergencia COVID-19
}

\author{
Mag. Carol Beatriz Bao Ratzemberg \\ cbaor@ucvvirtual.edu.pe \\ Escuela de posgrado \\ Universidad César Vallejo \\ ORCID: 0000-0003-4205-7446 \\ José Manuel Delgado Bardales \\ jmdelgadob@ucvvirtual.edu.pe \\ Escuela de posgrado \\ Universidad César Vallejo \\ ORCID:0000-0001-6574-2759 \\ Scopus autor ID: 24070333700 \\ Código Renacyt: P0050554
}

\section{RESUMEN}

El contexto climático, social y ambiental que enfrentamos actualmente como sociedad y como academia multidisciplinaria, demanda una articulación con las nuevas tendencias en gestión pública y gobernanza ciudadana enfocadas en el análisis territorial para la toma de decisiones públicas y gestión por procesos para resultados positivos. La asimilación conceptual en la enseñanza universitaria permitirá preparar y entrenar a profesionales de las diferentes carreras en una visión social - ambiental y económica más participativa y consciente del impacto de las políticas públicas y megas tendencias en las macroeconomías de los mercados. Los artículos correlacionan con el enfoque de prioridades entorno al concepto de gobernanza, que se refiere a la capacidad de resolución eficaz, eficiente y oportuna de los asuntos públicos, rápidamente los ámbitos conceptuales comunes con el planteamiento de la descentralización del Estado para la capacidad del gasto en tiempos de emergencia COVID-19 con rigurosidad a los procesos y ser eficientes, efectivos en el gasto, por lo que existe una crisis ambiental global y de inequidad social con líderes en gestión del cambio y práctica del desarrollo, con gerentes capacees de resolver la crisis aprovechando las oportunidades y proponiendo soluciones de bienestar económico con equidad social, sostenibilidad y sustentabilidad.

Palabras clave: Gobernanza, análisis territorial, emergencia, covid-19. 


\title{
Governance with territorial analysis in the COVID-19 Emergency
}

\begin{abstract}
The climate, social and environmental context that we currently face as a society and as a multidisciplinary academy, demands an articulation with the new trends in public management and citizen governance focused on territorial analysis for public decision-making and management by processes for positive results. The conceptual assimilation in university education will allow to prepare and train professionals from different careers in a more participatory social, environmental and economic vision, aware of the impact of public policies and mega trends in the macroeconomics of the markets. The articles correlate with the priority approach around the concept of governance, which refers to the capacity for effective, efficient and timely resolution of public affairs, quickly the common conceptual areas with the approach of the decentralization of the State for the spending capacity in times of COVID-19 emergency with rigorous processes and be efficient, effective in spending, so there is a global environmental crisis and social inequity with leaders in change management and development practice, with managers capable of solving the crisis, taking advantage of opportunities and proposing solutions for economic well-being with social equity, sustainability and sustainability.
\end{abstract}

Keywords: Governance, territorial analysis, emergency, covid-19

Artículo recibido: 06 oct. 2020 Aceptado para publicación: 14 nov. 2020 Correspondencia: cbaor@ucvvirtual.edu.pe, Conflictos de Interés: Ninguna que declarar 


\section{INTRODUCCIÓN}

Generalmente, en la gestión pública se busca un entorno libre de riesgos en los ámbitos de la salud pública, el ambiente, el crecimiento sostenible y otros. Sin embargo, esta ausencia de riesgo resulta ser complicado en un contexto donde la propia participación con el entorno implica la susceptibilidad a recurrencia de eventos que afecten su bienestar. El concepto de riesgo no tiene un consenso claro en la literatura. Por un lado, no hay duda que, en el uso común, en términos del lenguaje, el riesgo tiene un significado intuitivo, pues éste se entiende como una situación que implica enfrentar una actividad causante de consecuencias indeseables e inesperadas. Por otro lado, el concepto toma acepciones diferentes cuando comienza a ser utilizado en contextos científicos (Coy, 2010). Desde un punto de vista del positivismo (el positivismo es el pensamiento que afirma que el conocimiento auténtico es el conocimiento científico, el cual se deriva del contraste de hipótesis a través del método científico) moderno, el riesgo es la probabilidad de un evento adverso, el cual trae consecuencias igual de adversas. Por su parte, el constructivismo (el constructivismo afirma que el conocimiento es una construcción del ser humano: cada persona percibe la realidad, la organiza y le da sentido en forma de constructos, lo cual contribuye a dar sentido y unicidad de la realidad (Ortiz D, 2015) social considera que el riesgo es la percepción subjetiva formada por los filtros culturales y la estructura social (Rosa, 2003).

Tanto la visión positivista como la subjetivista constituyen puntos de vista de extremos, los cuales, si consideran el futuro como predeterminado o independiente a las actividades humanas, el término de riesgo no tendría algún sentido. Si la capacidad de identificar, medir y comprender los riesgos (abarcar desde lo supuestamente seguro hasta lo totalmente incierto) está limitada, el riesgo será cada vez más un estado menos objetivo del mundo, y por tanto una construcción más social (Rosa, 2003). De acuerdo a lo anterior, los riesgos no están determinados sólo por el estado objetivo del riesgo (probabilidad), sino también relacionados a las creencias, actitudes, juicios y sentimientos del individuo, además de su disposición a factores sociales, culturales y políticos (Rosa, 2003; Micic, 2016). Este enfoque proveniente de las ciencias sociales, no solo se basa en las fuentes del peligro o en los aspectos de las cuantificación o modelamiento de la magnitud de los daños, sino más bien en la inclusión social de los peligros y en el manejo de los riesgos (Coy, 2010). Además, Aven y Renn (2009) definen el riesgo como una situación o evento incierto, el cual se relaciona con la gravedad de los eventos y las consecuencias de una actividad (resultados inciertos) con respecto a algo que los humanos valoran.

La definición anterior muestra dos componentes o dimensiones: la incertidumbre y el impacto (Frame, 2003). Al incorporar la dimensión de incertidumbre en el concepto de riesgo, éstos son susceptibles a ser comparados, de manera que es posible considerarlos altos o bajos. Para esto, la herramienta básica son las probabilidades, las cuales vienen a ser la medida de dicha incertidumbre 
relacionada con algún evento; por ejemplo, el riesgo asociado a un ataque terrorista. De acuerdo a Aven y Renn (2009), el riesgo en este caso se refiere a la incertidumbre y la gravedad de las consecuencias de un ataque terrorista. Sin embargo, para especificar la probabilidad un riesgo, los datos estadísticos son insuficientes. En estos casos es posible el uso de una expresión cualitativa del conocimiento sobre los fenómenos y los procesos subyacentes (Aven y Renn, 2009). De esta manera, según Aven (2003), las "probabilidades subjetivas" no son estimadas sino asignadas, y expresan incertidumbre bajo el juicio de analistas o expertos respecto a algunos eventos o cantidades. El WEF (2018) en "The Global Risk" basa su evaluación en encuestas aplicadas a su amplia red de negocios que se extiende entre empresarios, gobiernos, sociedad civil y líderes de opinión del Foro a nivel mundial. El marco metodológico seguido por el WEF (2018) es similar al propuesto por Lee (1981), donde el "riesgo global" es definido como un evento o condición incierta que, si ocurre, puede causar un impacto negativo significativo para varios países o industrias en los próximos 10 años.

Un "Riesgo global" es un evento o condición incierta que, de ocurrir, podría generar un impacto negativo al bienestar de la población peruana dentro de los próximos 12 años. De la definición anterior se puede observar que es consistente con las definiciones Aven y Renn (2009) y Rosa (2003), incluyendo además un espacio temporal específico (12 años) y una población determinada (población peruana). Estos aspectos de la definición de riesgo permiten al experto enmarcar de manera más sencilla su análisis de impacto, desde un enfoque cualitativo de acuerdo a Frame (2003), y probabilidad, desde una perspectiva subjetiva como lo señala Aven y Renn (2009). Una oportunidad es un evento o entorno global que favorece un proceso de construcción de futuro deseable, siempre que se tomen las decisiones pertinentes para su aprovechamiento. Los riesgos pueden tener dos puntos básicos que influyen en su definición. El primero, es el contexto en el que se presenta la posibilidad de riesgo. El segundo es la percepción de riesgo por parte de los responsables de estos procesos. Esto implica que el riesgo es la consecuencia de estar o enfrentar una situación, actividad o escenario de peligro, el cual es el causante de consecuencias indeseables inesperadas o adversas (Martínez, 2002). Lo mismo se puede decir de las oportunidades las cuales también tienen una probabilidad de ser aprovechadas, así como una percepción de magnitud positiva.

El informe de análisis de futuro del CEPLAN presenta 14 megatendencias, los cuales son pertinentes analizar junto a los riesgos; dado que generarán cambios simultáneos en varios aspectos de la sociedad. A continuación, se presentan las 14 megatendencias: T1. Cambio climático T2. Degradación del ambiente T3. Empoderamiento del ciudadano T4. Inestabilidad de la gobernanza global T5. Desigualdad de la riqueza e ingresos T6. Multipolaridad del poder T7. Crecimiento de ciudades T8. Ciberdependencia T9. Interconectividad T10. Envejecimiento de la población T11. 
Incremento de las clases medias T12. Convergencia tecnológica T13. Movilidad geográfica T14. Enfermedades crónicas degenerativas. El Perú enfrenta un escenario global profundamente complejo. Generalmente, esta complejidad es ajena al control del país, y muestra que es sumamente vulnerable a los riesgos que ocurren en el contexto internacional, ya sean económicos, ambientales, sociales o tecnológicos. De igual manera, los escenarios globales ofrecen oportunidades que dependiendo de las acciones que se tomen, pueden generar impactos positivos sobre el bienestar de la población peruana.

Por lo mencionado no propusimos como problema: ¿Cuáles son los principales riesgos y oportunidades a los que se enfrenta el Perú en los próximos doce años para la gobernanza y la gobernabilidad? El Centro Nacional de Planeamiento Estratégico (CEPLAN) ha analizado los riesgos más importantes en un momento de incertidumbre global donde el contexto político, económico, social y tecnológico cambia a pasos acelerados; así como también las oportunidades que favorezcan la construcción de un futuro deseado al 2030, siempre que se tomen las decisiones pertinentes para el aprovechamiento óptimo de las mismas. En ese sentido, el presente estudio ha identificado una lista de 27 riesgos y 28 oportunidades a partir de: (i) los reportes "Global Risk" del Foro Económico Mundial (WEF por sus siglas en inglés) y el "Global Opportunity Report" de DNV-GL; y (ii) de los PESEM de catorce ministerios del Perú. De esta lista, se ha realizado una consulta técnica dirigida a expertos en prospectiva a fin de priorizar los principales riesgos y oportunidades que podrían afectar a Perú al 2030. Metodológicamente, tanto los riesgos y oportunidades partieron de consultas de expertos, mediante las cuales se les asignaron una probabilidad de ocurrencia, así como la magnitud de sus impactos (negativos en el caso de los riesgos, y positivos en el caso de las oportunidades). Asimismo, se analizaron las relaciones con las mega tendencias de manera separadas, y posteriormente se detalló el análisis de los principales riesgos y oportunidades.

Las oportunidades no se encuentran aisladas. Estas interactúan entre ellas, con las tendencias y megatendencias dentro del plano de los escenarios de futuro. En ese sentido, al igual que el análisis de riesgos globales, la figura 01 muestra las interrelaciones entre las oportunidades y las megatendencias. En contraste a los riesgos globales, la red de oportunidades globales no distingue un grupo particular que se encuentre aislado de la red de conexiones. En ese sentido, tanto las oportunidades ambientales, económicas, tecnológicas y sociales se encuentran interconectadas con diversas megatendencias, especialmente las económicas que denotan mayor dispersión dentro de la red. 


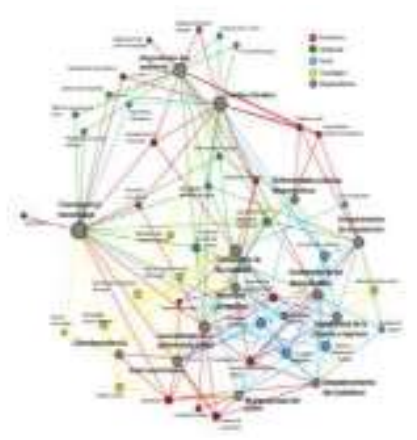

Figura 01: Interconexiones de oportunidades y megatendencias con la red.

Nota. El tamaño de los nodos (círculos) de las metagentencias (color gris) está asociado al nivel de relación con las oportunidades, de manera que un nodo con mayor tamaño representa más oportunidades asociados a él. Elaboración CEPLAN a partir de los resultados de la consulta técnica realizada. (CEPLAN análisis de riesgos y oportunidades al 2030- 2019).

El análisis de los vínculos de las oportunidades con las megatendencias revela la estrecha relación que tienen las oportunidades con la convergencia tecnológica. En este caso, esta mega tendencia es la que más se relaciona con las oportunidades identificadas, lo cual se denota en el número de aristas que convergen en su nodo. Esto se asocia con el pronto inicio de la denominada Cuarta Revolución Industrial, término propuesto por el WEF que describe una nueva era donde los sistemas ciberfísicos permitirán desarrollar nuevas capacidades para las personas y máquinas (Davis, 2016). En ese sentido, se desarrollan las siguientes oportunidades: (i) Tecnología móvil e internet, (ii) Replantear el uso del plástico, (iii) Cadena de bloques (blockchain), (iv) Demanda de productos orgánicos, (v) Uso de energía solar y eólica, y (vi) Uso de equipos de alta tecnología. Desde hace mucho tiempo en el Perú, la ciudadanía exige un mejor Estado, presente, activo y efectivo en todo el territorio nacional. A pesar de que en los últimos años la recaudación fiscal y el presupuesto público se han incrementado sustancialmente, las entidades públicas siguen teniendo una limitada capacidad de gestión, lo cual les impide proveer con eficacia, eficiencia y transparencia servicios públicos de calidad a los ciudadanos. Buscando acercar el Estado a los ciudadanos más homogéneamente en todo el país, hace una década se inició un profundo proceso de transformación, que significó la transferencia de funciones y recursos desde el nivel central a los gobiernos regionales y locales para que, en ejercicio de su autonomía política, económica y administrativa sirvieran de manera más cercana y efectiva a la ciudadanía. El proceso de descentralización satisfizo aspiraciones y necesidades largamente postergadas, y también ha generado nuevas expectativas por mayor inclusión y oportunidades para el desarrollo. Sin embargo, esta transferencia de funciones y el incremento de recursos no han sido acompañados por mejores capacidades descentralizadas de gestión. Iniciar el proceso de descentralización en un contexto como el antes descrito ha significado que las limitaciones mostradas por las entidades del Gobierno Central se vean reproducidas a nivel 
descentralizado. Como consecuencia de ello, las entidades públicas nacionales, regionales y locales no muestran en general, tener las capacidades de gobierno y de gerencia suficientes para proveer más y mejores bienes y servicios públicos a todos los ciudadanos en todo el país, según su necesidad. El Perú tiene uno de los Estados más disfuncionales de América Latina. Muchas instituciones estatales no funcionan, y en algunas zonas, ni siquiera existen. Nuestro sistema no puede mostrarse ajeno a los grandes cambios que se están enfrentando día a día para alcanzar estándares de calidad. Una de sus metas principales deberá ser elevar el buen servicio a la ciudadanía y a la responsabilidad social con interculturalidad y género. En muchas partes del interior de país, los servicios públicos (educación, salud, agua potable, infraestructura) simplemente no existen, y en caso "existieran", se encuentran plagados por la corrupción y la ineficiencia en su gestión. Los gobiernos locales y regionales carecen de una adecuada capacidad administrativa. A este contexto se suma la apreciación pública de que la seguridad y la justicia - funciones básicas del Estado - no son las adecuadas y se encuentran mínimamente garantizadas por quienes las ejercen.

Las deficiencias del Estado Peruano, tienen un alto impacto en la vida de los ciudadanos y en las actividades empresariales, lo cual degenera la sensación de bienestar en el común de la población, en la competitividad empresarial, en la legitimidad de los gobiernos y por lo tanto, en el sistema democrático y sus instituciones. Tomando en cuenta las consecuencias que tienen las deficiencias de desempeño del Estado en la vida de las personas y en la competitividad y gobernabilidad democrática del país, es que se requiere con urgencia de una política integradora de modernización en la gestión pública, para asegurar que las entidades que conforman los tres niveles de gobierno actúen de manera articulada y consistente, direccionando sus esfuerzos a un mejor desempeño general del

Estado, sus políticas y forma de gestionar el país al servicio de los ciudadanos. Como se ha mencionado hasta el momento, existen graves deficiencias y desarticulaciones en la prestación actual de los servicios públicos, esta problemática incide de manera negativa en la percepción ciudadana sobre la gestión pública y el desempeño del Estado en el Perú. Frente a esta situación, ¿cuáles son los principales problemas de gestión pública que deben resolverse con urgencia?, Del "Documento de Identificación de la Problemática y Mapeo de Actores" se puede identificar lo siguiente: 1. Ausencia de un sistema eficiente de planeamiento y problemas de articulación con el sistema de presupuesto público; No queda claro el rol efectivo del CEPLAN, ni el de las políticas u objetivos prioritarios del Gobierno. Asimismo, se han identificado problemas en la definición de los objetivos -los cuales, no necesariamente recogen las demandas de la población, esto conlleva a que las brechas que se pretenden cubrir no se encuentran estimadas de manera adecuada. Además, no se ha logrado asegurar un correcto alineamiento entre las políticas públicas nacionales y sectoriales con las políticas territoriales (responsabilidad de los gobiernos descentralizados), así como tampoco 
se han logrado articular de manera correcta los Planes de Desarrollo Concertado (PDC), los Planes Estratégicos Institucionales (PEI), los Planes Operativos Institucionales (POI), ni una correcta vinculación de éstos con los documentos de gestión pública y los programas presupuestales. Todo lo descrito, cuando es estudiado en la práctica, no logra utilizar el planeamiento como una herramienta efectiva de gestión y no logra articular el plan y el presupuesto público. Otro aspecto a resaltar es que existen limitaciones en cuanto a las capacidades de las entidades para identificar y priorizar programas y proyectos de envergadura y alto impacto en la calidad de vida de los ciudadanos. Cuando evaluamos el presupuesto público, a pesar de haberse logrado algunos avances en su concepción y formulación a través de la implementación progresiva del presupuesto para resultados más eficientes; se puede encontrar aún, que existen graves problemas pendientes; en ciertos sectores persiste una asignación inercial de recursos (monto asignado el año anterior y negociado con el MEF por incrementos).

Asimismo, debe mencionarse que el gobierno nacional tiene el 70\% del gasto corriente total, las regiones el 19\% y los gobiernos locales, el 11\%. Adicionalmente, existe poca predictibilidad de los recursos transferidos como consecuencia del canon y las regalías. Además, no siempre la asignación de los recursos responde a una definición clara de las prioridades del país y a una objetiva determinación de metas, de productos o servicios que cada entidad debe ofrecer, así como de sus respectivos costos. Ello hace que en el nivel operativo no necesariamente exista coherencia entre los insumos que van a ser adquiridos y los servicios que serán provistos con ellos, con lo cual, los presupuestos no siempre se dirigen a cerrar las brechas o déficits existentes de infraestructura y servicios públicos, ni responden a las prioridades ciudadanas.

Deficiente diseño de la estructura de organización y funciones: En muchas instituciones públicas su estructura de organización y funciones (agrupamiento de actividades y asignación de responsabilidades dentro de la entidad) no necesariamente viene siendo congruente con las funciones que deben cumplir, ni tampoco con los objetivos que puedan haberse fijado como resultado de sus procesos de planeamiento estratégico-operativo y de su presupuestario. Ello puede deberse a que las organizaciones fueron diseñadas bajo un modelo de gestión funcional, con estructuras jerárquicas, estamentales y sin claridad en los procesos que deben realizar para entregar los bienes y servicios públicos de su responsabilidad con la calidad y pertinencia requeridos. Además, los lineamientos y los modelos vigentes para la formulación de documentos de gestión ROF, CAP, etc.- imponen normas uniformes de organización para la gran diversidad de entidades existentes, en especial en el caso de los Gobiernos Regionales, para los que su Ley Orgánica establece un modelo único y demasiado detallado de organización que no considera la heterogeneidad de las realidades regionales. 
El origen del concepto de gobernanza no es claro y ha sido desarrollado por muy diversas escuelas de pensamiento, desde la corriente neoliberal de la nueva gerencia pública, hasta el neoinstitucionalismo de un nuevo estado social o el universalismo comunitario de la sociedad civil. Siguiendo a Farinós Dasí (2008), el término es tomado inicialmente por la economía institucional y de regulación como mecanismo para simplificar los procesos de regulación y de intervención de los actores públicos, y facilitar así la toma de decisiones del resto de los agentes sociales.

El Banco Mundial entiende la gobernanza como el conjunto de procesos e instituciones a través de las cuales se determina la forma en que se ejerce el poder de un país y se lo vincula, principalmente, con el buen gobierno, término que refiere directamente a la transparencia, y a la eficacia en la elección y el control de los gobernantes, la capacidad de la administración para gestionar los recursos, la participación ciudadana y la garantía del Estado de derecho. Esta definición ha sido criticada desde diferentes enfoques porque reduce la gobernanza a una "herramienta ideológica para una política de Estado mínimo" (Rosas-Ferrusca, Calderón- Maya y Campos-Alanís, 2012: 115). A pesar del carácter polisémico del concepto (Farinós Dasí, 2008), el rasgo común en la diversidad de acepciones y connotaciones en torno a la idea de gobernanza es la redefinición del rol del Estado y sus relaciones con la sociedad civil. Autores como Gantus (2013) y Farinós Dasí (2008) sostienen que el Estado moderno, manejado por las clases políticas tradicionales, tiene serias dificultades para dar respuestas a las demandas sociales en un contexto de creciente corrupción, falta de transparencia y crisis de la representatividad, lo que explica las demandas de mayor participación, democratización y apertura que hacen muchos actores al Estado. De acuerdo con esto, la dirección y la determinación de los objetivos de la sociedad ya no es una función exclusiva del gobierno, sino producto de una multiplicidad de actores que configuran nuevas relaciones y que están reclamando el tránsito de un control jerárquico ejercido desde y por el Estado, hacia formas más horizontales y cooperativas. En otras palabras, la gobernanza promueve un estilo de gobierno sustentado más en la coordinación de intereses de diversos actores, que en el mando formal y normativo propiciado por y desde el Estado.

El estudio se propuso como problema. ¿Cuáles son las características de la gobernanza con análisis territorial en Emergencia COVID-19? Como objetivo se propuso: Determinar las características de la gobernanza con análisis territorial en Emergencia COVID-19.

\section{ESTRATEGIAS METODOLÓGICAS O MATERIALES Y MÉTODOS}

El tipo es cualitativo y el diseño de estudio es una revisión sistemática, las revisiones sistemáticas son investigaciones científicas en las cuales la unidad de análisis son los estudios originales primarios, constituyen una herramienta esencial para sintetizar la información científica disponible, incrementar la validez de las conclusiones de estudios individuales e identificar áreas de incertidumbre donde sea necesario realizar investigación. 
La recolección de datos se realizó a través de la revisión bibliográfica de artículos de investigaciones tanto nacionales como internacionales que tuvieron como tema principal gobernanza ciudadana con análisis territorial de los riesgos y oportunidades; de todos los artículos que se encontraron, se incluyeron los más importantes según nivel de evidencia y se excluyeron los menos relevantes. Se estableció la búsqueda siempre y cuando se tuvo acceso al texto completo del artículo científico. El análisis de la revisión sistemática evaluando cada uno de los artículos para una comparación de los puntos o características en las cuales concuerda y los puntos en los que existe discrepancia entre artículos nacionales e internacionales. Además, de acuerdo a criterios técnicos pre establecidos, se realizó una evaluación crítica e intensiva de cada artículo, a partir de ello, se determinó la calidad de la evidencia y la fuerza de recomendación para cada artículo creando una nueva perspectiva teórica en el presente artículo. 


\section{RESULTADOS Y DISCUSIÓN}

\section{Resultados}

\section{DATOS DE LA PUBLICACIÓN}

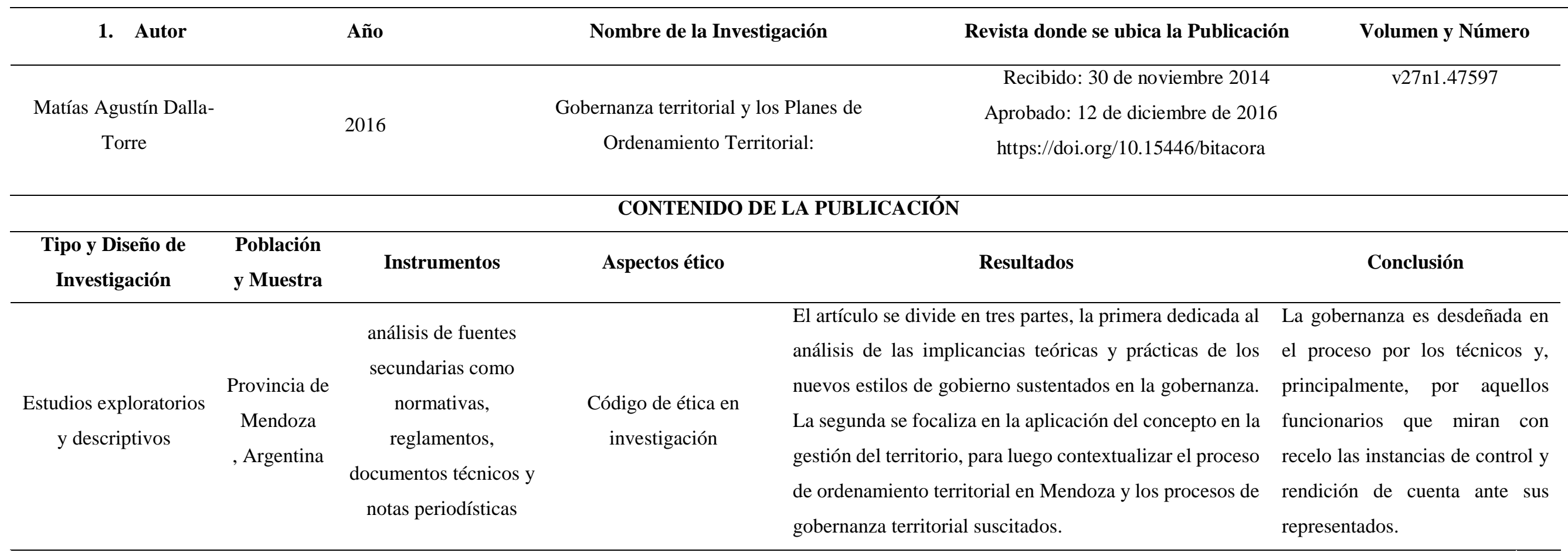

\section{DATOS DE LA PUBLICACIÓN}

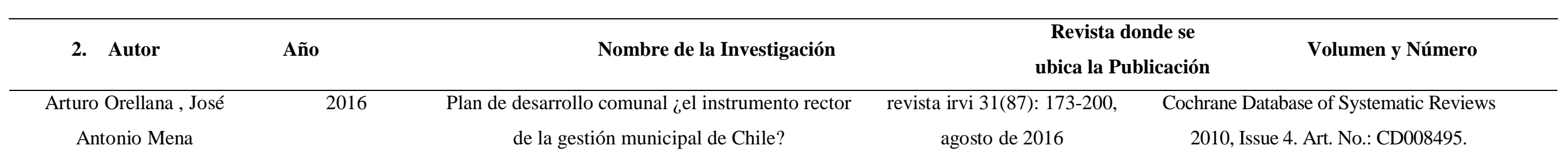

Ciencia Latina Revista Científica Multidisciplinar, Ciudad de México, México. 


\section{CONTENIDO DE LA PUBLICACIÓN}

\begin{tabular}{|c|c|c|c|c|}
\hline $\begin{array}{l}\text { Tipo y Diseño } \\
\text { de } \\
\text { Investigación }\end{array}$ & $\begin{array}{c}\text { Población y } \\
\text { Muestra }\end{array}$ & Instrumentos & Aspectos ético & Conclusión \\
\hline $\begin{array}{l}\text { Cualitativo } \\
\text { de revisión } \\
\text { sistemática }\end{array}$ & Documentos & $\begin{array}{l}\text { análisis de } \\
\text { contenido a } \\
\text { partir de una } \\
\text { muestra de } 58 \\
\text { documentos }\end{array}$ & $\begin{array}{l}\text { El resultado de este trabajo refleja una } \\
\text { situación bastante desalentadora, ya que en la } \\
\text { mayoría de las comunas el cumplimiento de } \\
\text { los compromisos establecidos en el desarrollo } \\
\text { del PLADECO resulta ser inferior al } 50 \% \text { en } \\
\text { todos los ejes de análisis, a excepción de } \\
\text { accesibilidad. }\end{array}$ & $\begin{array}{l}\text { Se debe emprender modificaciones de orden técnico y } \\
\text { legal para que este instrumento PLADECO sea realmente } \\
\text { un instrumento rector de la gestión municipal en Chile. }\end{array}$ \\
\hline \multicolumn{5}{|c|}{ DATOS DE LA PUBLICACIÓN } \\
\hline 3. Autor & Año & & Nombre de la Investigación & $\begin{array}{c}\text { Revista donde se } \\
\text { ubica la Publicación }\end{array}$ \\
\hline Arturo Orellana, & 2016 & & Plan de desarrollo comunal ¿el instrumento rector de la gestión & Cochrane Database of Systematic \\
\hline José Antonio & & & municipal de Chile? & Reviews 2010, Issue 4. Art. No.: \\
\hline Mena & & & & CD008495 \\
\hline \multicolumn{5}{|c|}{ CONTENIDO DE LA PUBLICACIÓN } \\
\hline $\begin{array}{l}\text { Tipo y Diseño de } \\
\text { Investigación }\end{array}$ & $\begin{array}{c}\text { Población y } \\
\text { Muestra }\end{array}$ & Instrumentos & Aspectos ético & Conclusión \\
\hline $\begin{array}{l}\text { Cualitativo } \\
\text { de revisión } \\
\text { sistemática }\end{array}$ & Documentos & $\begin{array}{l}\text { análisis de } \\
\text { contenido a } \\
\text { partir de una }\end{array}$ & $\begin{array}{l}\text { El resultado de este trabajo refleja una } \\
\text { situación bastante desalentadora, ya que en la } \\
\text { mayoría de las comunas el cumplimiento de }\end{array}$ & $\begin{array}{l}\text { Se debe emprender modificaciones de orden técnico y } \\
\text { legal para que este instrumento PLADECO sea realmente } \\
\text { un instrumento rector de la gestión municipal en Chile. }\end{array}$ \\
\hline
\end{tabular}

Ciencia Latina Revista Científica Multidisciplinar, Ciudad de México, México. 


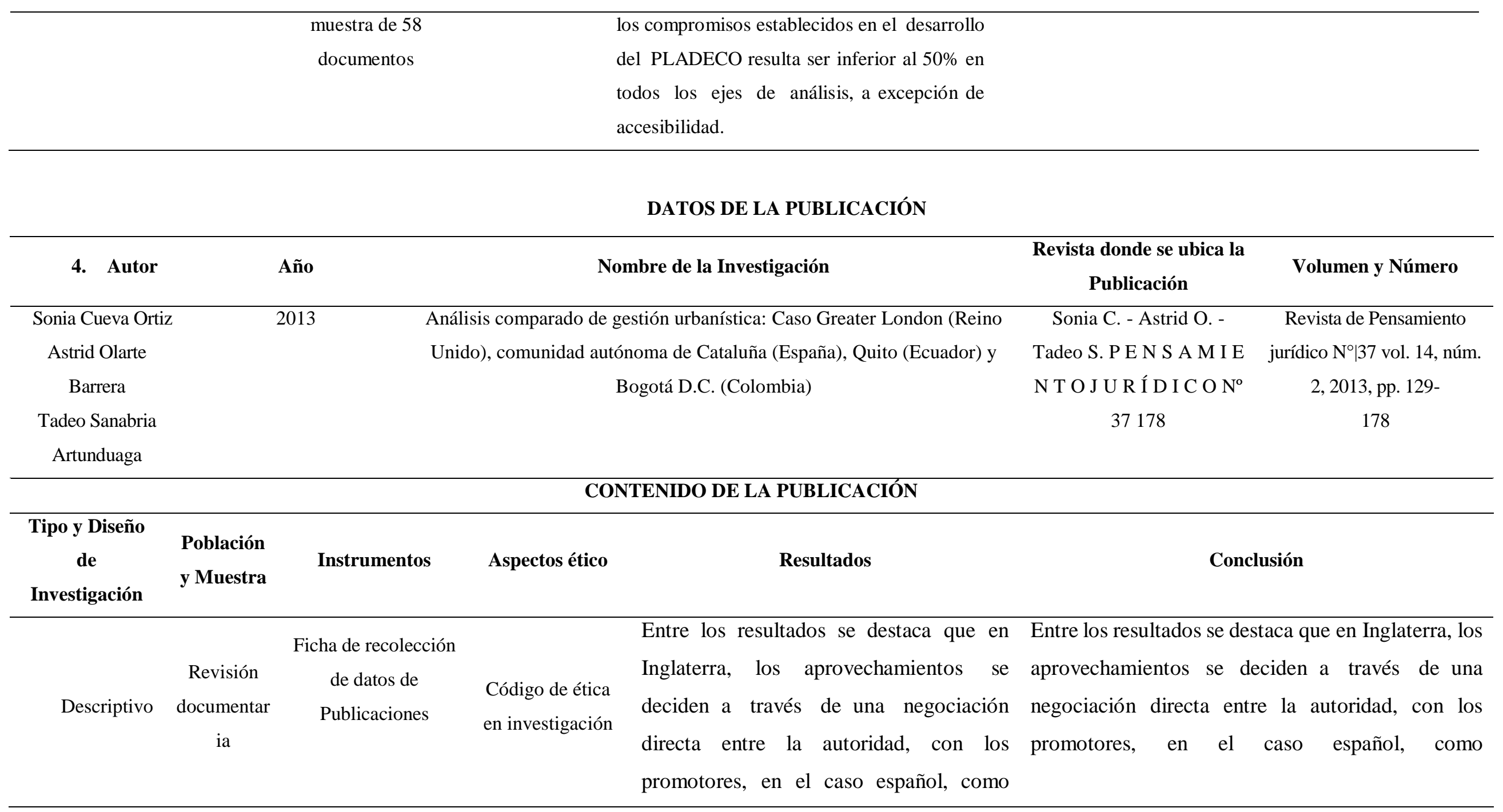

Ciencia Latina Revista Científica Multidisciplinar, Ciudad de México, México.

ISSN 2707-2207 / ISSN 2707-2215 (en línea), julio-diciembre, 2020, Volumen 4, Número 2. https://doi.org/10.37811/cl_rcm.v4i2.119 p. 813 


\section{DATOS DE LA PUBLICACIÓN}

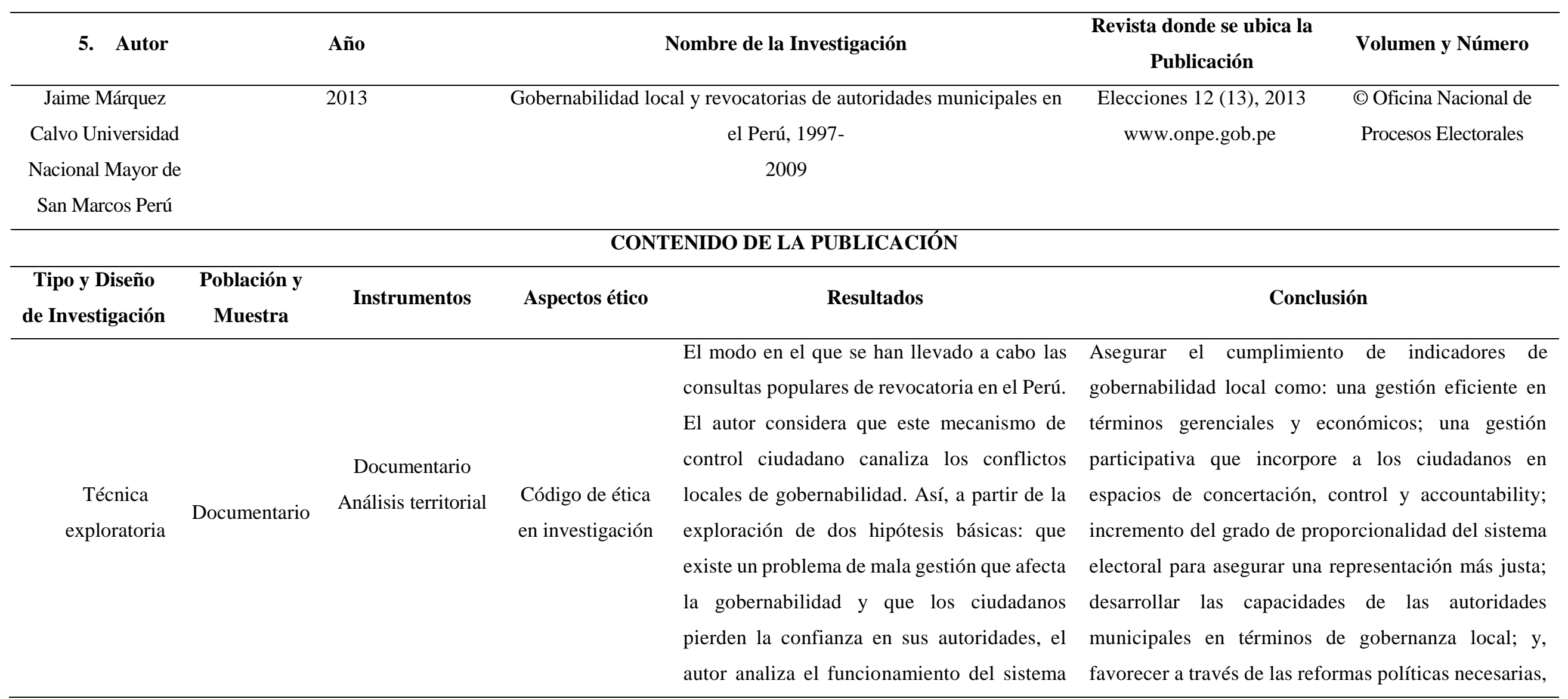

Ciencia Latina Revista Científica Multidisciplinar, Ciudad de México, México. 
electoral y del sistema político local con la

finalidad de comprender la relación entre los

conflictos sociales que surgen en el ámbito

local y los procesos de control y de consulta

que son promovidos por los ciudadanos la capacidad de los actores políticos locales para la formación de coaliciones que den sostenibilidad futura a los gobiernos locales

\section{DATOS DE LA PUBLICACIÓN}

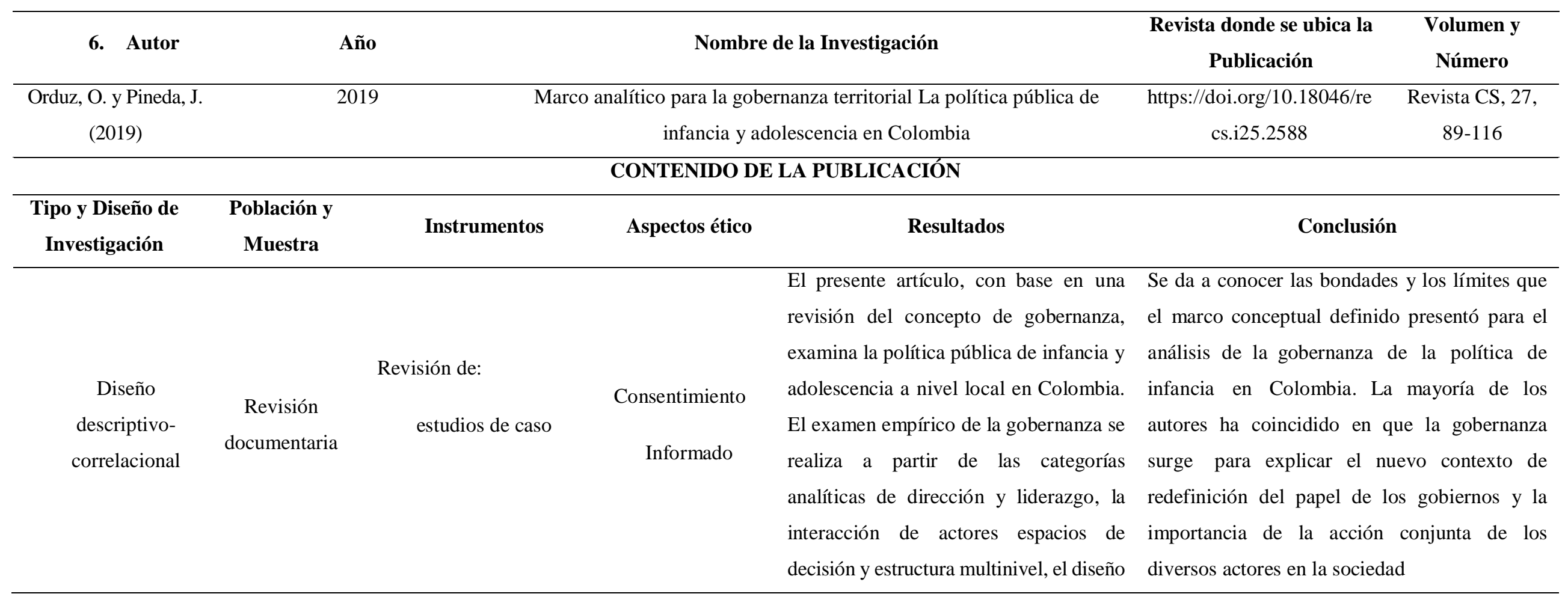

Ciencia Latina Revista Científica Multidisciplinar, Ciudad de México, México.

ISSN 2707-2207 / ISSN 2707-2215 (en línea), julio-diciembre, 2020, Volumen 4, Número 2. https://doi.org/10.37811/cl_rcm.v4i2.119 p. 815 


\section{DATOS DE LA PUBLICACIÓN}

\begin{tabular}{|c|c|c|c|c|}
\hline 7. Autor & & Año & Nombre de la Investigación & $\begin{array}{c}\text { Revista donde se ubica la } \\
\text { Publicación }\end{array}$ \\
\hline María Claudia & \multicolumn{2}{|r|}{2017} & Más allá de la capacidad estatal: sociedad civil & https://dx.doi.org/10.744 \\
\hline Augusto Meléndez & & & e implementación de políticas a nivel & 0/colombiaint 90.2017 .04 \\
\hline Eduardo Dargent & & & subnacional en Cusco y San Martín (Perú) & $2017 \bullet$ pp. 99-125 \\
\hline \multicolumn{5}{|l|}{ Bocanegra Stéphani } \\
\hline \multicolumn{5}{|l|}{ Rousseau } \\
\hline \multicolumn{5}{|c|}{ CONTENIDO DE LA PUBLICACIÓN } \\
\hline $\begin{array}{c}\text { Tipo y Diseño } \\
\text { de } \\
\text { Investigación }\end{array}$ & $\begin{array}{l}\text { Población y } \\
\text { Muestra }\end{array}$ & Instrumentos & Aspectos ético & Conclusión \\
\hline Descriptivo & $\begin{array}{c}\text { Revisión } \\
\text { documentaria }\end{array}$ & $\begin{array}{l}\text { Ficha de } \\
\text { recolección de datos }\end{array}$ & $\begin{array}{cl} & \text { En este artículo presentamos dos casos que } \\
& \text { resaltan cómo la sociedad civil regional } \\
\text { Código de ética } & \text { contribuye a una mejor implementación de } \\
\text { de investigación } & \text { normas y políticas públicas en espacios } \\
& \text { subnacionales. En las regiones peruanas de } \\
& \text { San Martín y Cusco, actores de la sociedad } \\
& \text { civil aprovecharon el espacio de la }\end{array}$ & $\begin{array}{l}\text { Involucrar a la sociedad civil organizada y con } \\
\text { expertise en los temas de reforma, hacerla partícipe } \\
\text { de las políticas, o incluso diseñar políticas que } \\
\text { alineen los intereses del Estado con la sociedad, } \\
\text { sirven como garantía de continuidad. Nuestros } \\
\text { resultados apuntan a una causa por la que ciertas } \\
\text { competencias de gobiernos subnacionales se }\end{array}$ \\
\hline
\end{tabular}

Ciencia Latina Revista Científica Multidisciplinar, Ciudad de México, México. 
descentralización para poner en marcha de manera efectiva leyes nacionales (normas ambientales, en el primer caso, y derechos para la población hablante de idiomas indígenas, en el segundo). Estos casos ayudan a explicar la heterogeneidad en la implementación de las

normas en espacios subnacionales, un

fenómeno común en América Latina

desarrollan más que otras: una sociedad civil fuerte e interesada en los asuntos regulados en las normas será un buen predictor de este desarrollo. Resaltar esta relevancia de la sociedad civil para mejorar la implementación de leyes y políticas a nivel subnacional tiene implicancias teóricas y prácticas

\section{DATOS DE LA PUBLICACIÓN}

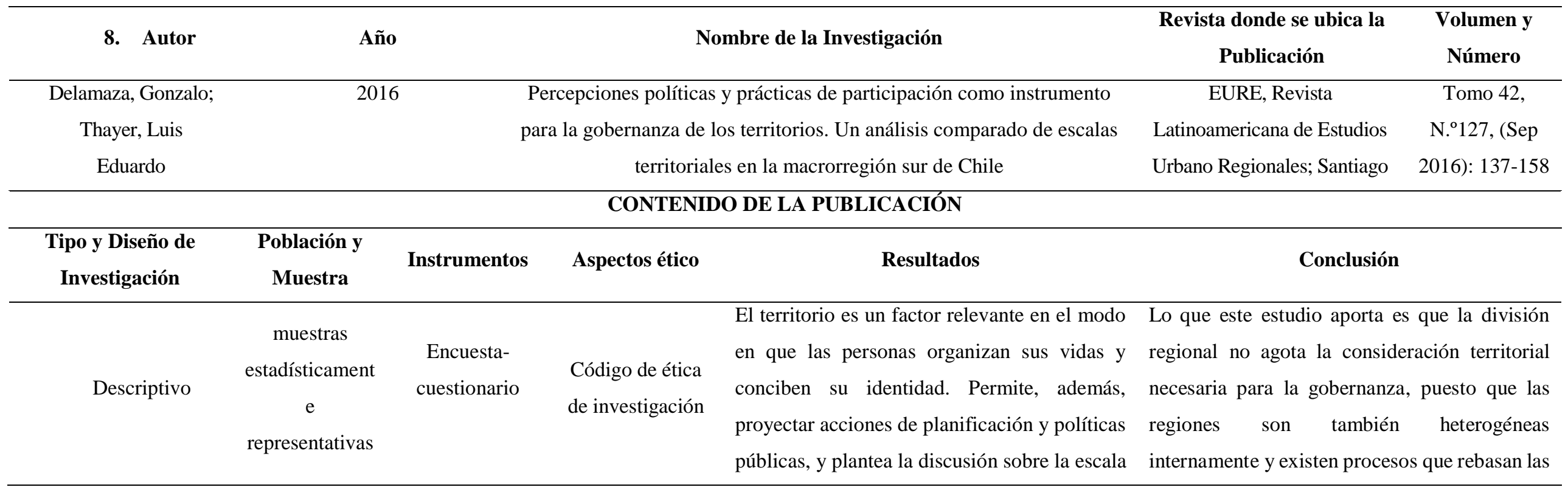

Ciencia Latina Revista Científica Multidisciplinar, Ciudad de México, México.

ISSN 2707-2207 / ISSN 2707-2215 (en línea), julio-diciembre, 2020, Volumen 4, Número 2. https://doi.org/10.37811/cl_rcm.v4i2.119 p. 817 
aplicada en el

año

2013 a tres

regiones

administrativas

en Chile:

Biobío, Los

Ríos y Los

Lago territorial más apropiada para esas acciones. El

presente artículo indaga en prácticas de la

participación ciudadana y en percepciones

sobre instituciones políticas, contrastando para

ello demarcaciones territoriales establecidas

administrativamente (regiones) y tipos de

territorio definidos según criterios

sociodemográficos (conurbación, ciudades

intermedias y zonas rurales). Con base en la conceptualización de gobernanza territorial, se presentan datos de una encuesta realizada en 2013, relativos a percepciones políticas y prácticas de participación de la ciudadanía, relacionadas con el espacio territorial. Se aborda así la definición y distinción entre territorios geográficos y su relación con la subjetividad ciudadana, como un componente relevante para la construcción de la gobernanza territorial y el despliegue de políticas públicas diferencias regionales $y$ afectan de similar manera a territorios ubicados en distintas regiones, que se acercan más a la noción de "territorios vividos". Ello es relevante en términos de conocimiento de la dinámica territorial del país, de construcción y ejercicio de la gobernanza -incluyendo las variables subjetivas que diferencian a las ciudadanas y ciudadanos en los territorios- y, finalmente, en el mejor desempeño de las políticas públicas, incluyendo el proceso de descentralización. Desde el punto de vista de la gobernanza democrática, no debiera orientarse necesariamente a redefinir las regiones y buscar un marco territorial distinto para potenciar políticas públicas basadas en una articulación entre Estado y sociedad. Debiera centrarse, más bien, en pensar las regiones atendiendo a su heterogeneidad territorial

Ciencia Latina Revista Científica Multidisciplinar, Ciudad de México, México.

ISSN 2707-2207 / ISSN 2707-2215 (en línea), julio-diciembre, 2020, Volumen 4, Número 2. https://doi.org/10.37811/cl_rcm.v4i2.119 p. 818 


\section{DATOS DE LA PUBLICACIÓN}

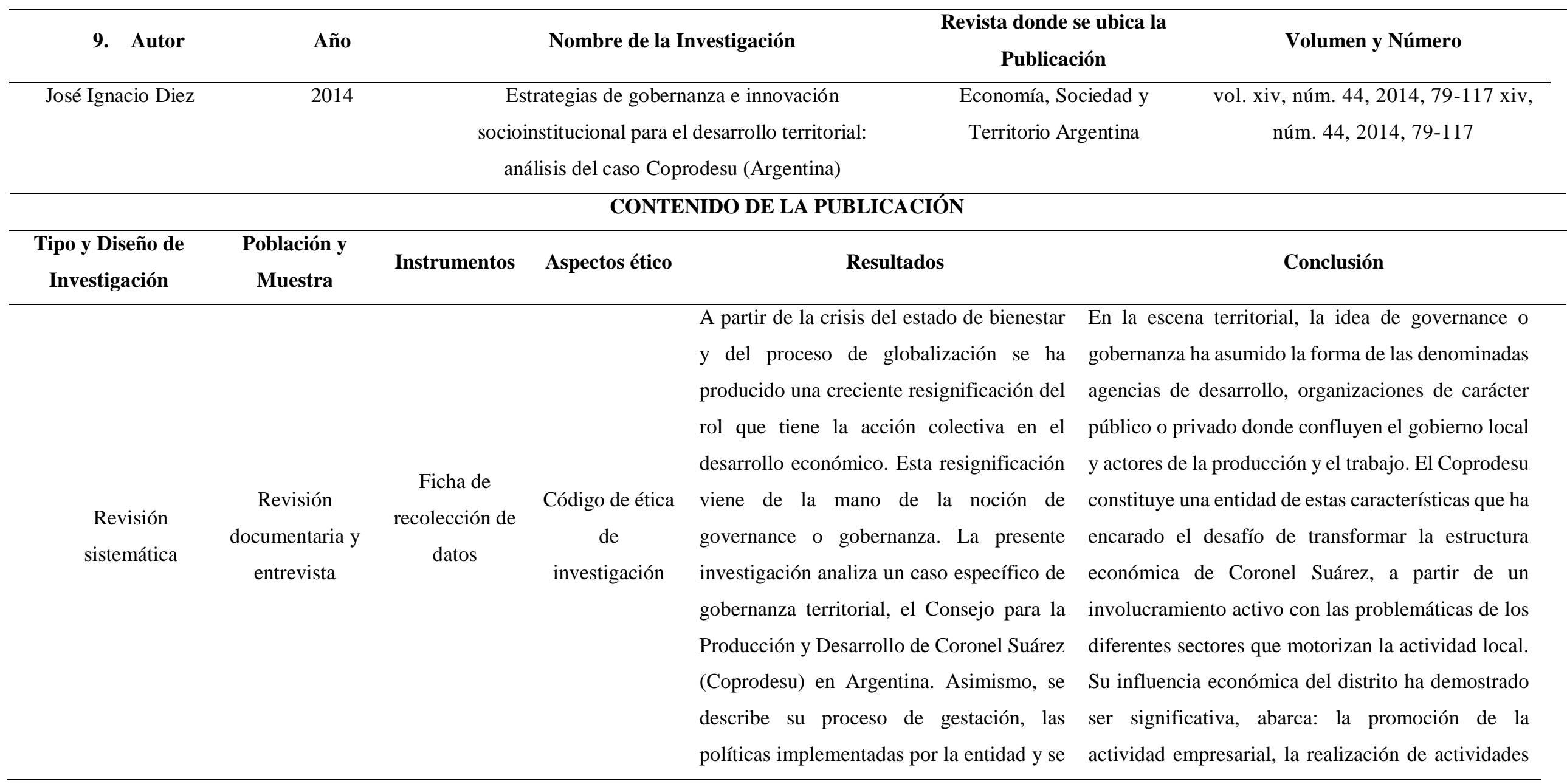

Ciencia Latina Revista Científica Multidisciplinar, Ciudad de México, México.

ISSN 2707-2207 / ISSN 2707-2215 (en línea), julio-diciembre, 2020, Volumen 4, Número 2.

https://doi.org/10.37811/cl_rcm.v4i2.119 p. 819 
identifican sus principales fortalezas y de capacitación, el otorgamiento de créditos y la

\section{DATOS DE LA PUBLICACIÓN}

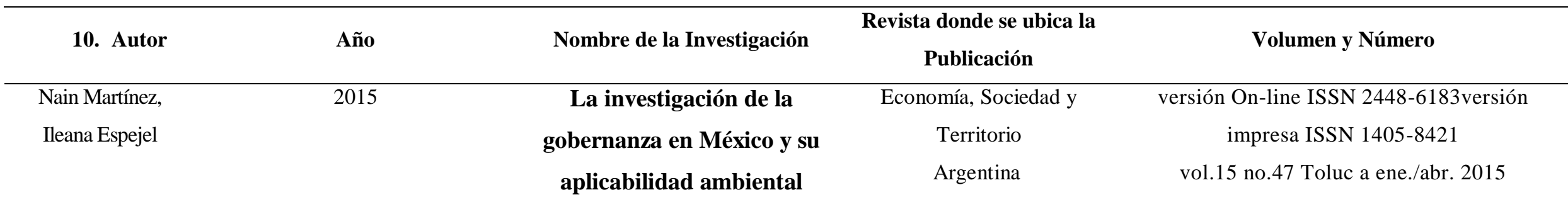

CONTENIDO DE LA PUBLICACIÓN

\begin{tabular}{|c|c|c|c|c|c|}
\hline $\begin{array}{c}\text { Tipo y Diseño de } \\
\text { Investigación }\end{array}$ & $\begin{array}{c}\text { Población y } \\
\text { Muestra }\end{array}$ & Instrume & Aspect & Resultados & Conclusión \\
\hline Descriptivo & $\begin{array}{c}75 \text { datos de } \\
60 \text { artículos de } \\
\text { investigación y } \\
15 \text { textos } \\
\text { provenientes de } \\
\text { libros (libros y } \\
\text { capítulos) }\end{array}$ & $\begin{array}{l}\text { Ficha de } \\
\text { recolección } \\
\text { de datos }\end{array}$ & $\begin{array}{c}\text { Código de } \\
\text { ética de } \\
\text { investigación }\end{array}$ & $\begin{array}{l}\text { La discusión sobre la gobernanza es cada vez más } \\
\text { frecuente dentro de las ciencias ambientales debido a } \\
\text { una mayor participación de los actores sociales en la } \\
\text { gestión ambiental. No obstante, las definiciones que } \\
\text { rodean el debate a menudo son poco claras. Se } \\
\text { contribuyó en la cohesión del debate al contextualizar } \\
\text { y clarificar los términos y definiciones pertinentes. } \\
\text { Con las publicaciones, se propone una nueva } \\
\text { definición de trabajo de gobernanza ambiental, al }\end{array}$ & $\begin{array}{l}\text { Finalmente, es previsible que en México la } \\
\text { investigación sobre la gobernanza ambiental } \\
\text { continúe acrecentándose en la medida en que se } \\
\text { analice la influencia de los procesos de interacción } \\
\text { entre los actores sociales y gubernamentales en el } \\
\text { sector ambiental. Por este motivo, realizar una } \\
\text { aportación para clarificar el uso de la teoría de la } \\
\text { gobernanza en este sector puede beneficiar a las } \\
\text { futuras investigaciones en la materia }\end{array}$ \\
\hline
\end{tabular}
distinguir este concepto del de gobernabilidad y gobierno 


\section{Discusión}

Posterior a la revisión sistemática de los artículos, del 100\%, el 54\% corresponden a España y Reino Unido, el 46\% a México, Colombia, Argentina, Chile, Bolivia y Perú. En relación a los diseños y tipos de estudios el 100\% son descriptivos.

Para conocer la gobernanza ciudadana debemos analizar las megatendencias de la globalización como ruta estratégica para determinar los riesgos y las oportunidades y así mejorar los procesos teniendo en cuenta el consenso de las PESEM(Plan Estratégico Sectorial Multianual) junto con CEPLAN que es el organismo técnico especializado que ejerce la función de órgano rector, orientador y de coordinación del Sistema Nacional de Planeamiento Estratégico; orientado al desarrollo de la planificación estratégica como instrumento técnico de gobierno y gestión para el desarrollo armónico y sostenido del país y el fortalecimiento de la gobernabilidad democrática en el marco del Estado constitucional de derecho pero es necesario tener en cuenta que la investigación está ligada a la innovación es por eso que la población humana en la comunidad como ciudadano del mundo no es constante, esta cambia a través de los años, crece a diferentes ritmos y dependiendo del espacio geográfico se aplican tres tipos de gestión: por procesos, por resultados y estratégicos para implementar reformas en el diseño institucional con argumentos de mayor eficiencia en la gestión del Estado por la vía de acortar procesos, disminuir procedimientos, aliviar burocracia; y de mayor calidad democrática, cercanía de las agencias gubernamentales con la ciudadanía y rendición de cuentas. A estos argumentos se ha ido sumando, en forma cada vez más expectante, el enfoque territorial del desarrollo, que busca y promueve la articulación de activos y actores territoriales para expandir sus oportunidades.

La gobernanza hace referencia a un nuevo estilo de gestión de los asuntos comunes, que implica una mayor coordinación entre los componentes del proceso de gobierno, de tal forma que no queda exclusivamente en manos del Estado; enfocándose en la búsqueda de alternativas a la organización y control jerárquicos. De esta forma cobra especial relevancia el papel regulador del mercado (políticas de privatización y desregulación), así como la puesta en marcha de formas cooperativas y horizontales de gestión social (autogobierno local, inclusión de la sociedad civil en la toma de decisiones, etc.) En conjunto, estos elementos se manifiestan en la firma de tratados internacionales de libre comercio, acuerdos internacionales en normas y certificaciones de seguridad, estándares de calidad y 
regulaciones medio ambientales, así como en empresas con cadenas de producción dispersas geográficamente, las cuales responden a través de productos adaptados por una parte a regiones y países y por otro lado a grupos de consumidores en distintas naciones.

Ciertamente, las señales son positivas y dan cuenta de un proceso en marcha, pero nada hace pensar que el camino será expedito y, más bien, se observa que la agenda de la descentralización a menudo se confronta con prácticas sectoriales y centralistas que se contraponen al despliegue de las capacidades territoriales. En efecto, así como se han impulsado reformas y se observa mayor compromiso con los procesos de descentralización, los estudios muestran las limitaciones: falta de diseños globales sobre atribuciones y responsabilidades de los distintos niveles del Estado, superposición de funciones y competencias, un diseño fiscal basado en transferencias más que en capacidad fiscal territorial e insuficiencia de capacidades en autoridades, profesionales y técnicos localizados en los niveles intermedios de gobierno. La situación es desafiante: agenda política pro descentralización en marcha y diversas limitaciones y restricciones que llaman a realizar una discusión seria sobre asuntos de gobernanza regional territorial.

Los artículos mencionados tienen correlación con el enfoque que las prioridades en torno al concepto de gobernanza, que se refiere a la capacidad de resolución eficaz, eficiente y oportuna de los asuntos públicos, rápidamente se encuentran ámbitos conceptuales comunes con el planteamiento de la descentralización del Estado. Esta asociación viene desde dos puntos de vista. Examinado desde arriba, el Estado centralista, auto centrado y jerárquico no responde a las necesidades de la sociedad actual, reclamándose procesos de desconcentración del poder.

La gobernanza incorpora esta dinámica de cambios de la sociedad, las evidentes restricciones estatales para ser el único actor en la gestión de las políticas, los imperativos de colaboración público privado para atender dilemas del desarrollo económico y social y los requerimientos de profundización democrática, transparencia y cooperación. Expresa la evolución del Estado moderno a una nueva realidad de co-producción de soluciones a asuntos de interés público y a un escenario de nuevas demandas ciudadanas, que ya no se localizan en el actor colectivo clásico, sino en actores sociales que participan en el marco democrático, que esperan eficiencia y calidad de la política pública y que se presentan más cercanos y exigentes de respuestas adecuadas a sus demandas y necesidades. Señala 
Aguilar (2006): “el corazón del concepto de gobernanza es un concepto descentralizado de la dirección de la sociedad que ya no puede ser dirigida (controlada), por un centro".

Dalla, M., concluye que la gobernanza es desdeñada en el proceso por los técnicos, y principalmente los funcionarios que miran con recelo as instancias de control y rendición de cuenta ante sus representados. Orellana, A., Mena, J., Marín, M., se debe emprender modificaciones de orden técnico y legal para que este instrumento PLADECO sea realmente un instrumento rector de la gestión municipal en Chile. Cueva, S; Olarte, A., Sanabria, T., más que una llamada a la reflexión para los legisladores de nuestros ordenamientos urbanísticos exige una política pública transversal que eduque en la cultura de la participación ciudadana y active de forma correcta las diferentes fuerzas que confluyen en el territorio y se materializan en el planeamiento y gestión urbana.

Márquez, J., asegurar el cumplimiento de indicadores de gobernabilidad local como: una gestión eficiente en términos gerenciales y económicos; una gestión participativa que incorpore a los ciudadanos en espacios de concertación, control y accountability; incremento del grado de proporcionalidad del sistema electoral para asegurar una representación más justa; desarrollar las capacidades de las autoridades municipales en términos de gobernanza local; y, favorecer a través de las reformas políticas necesarias, la capacidad de los actores políticos locales para la formación de coaliciones que den sostenibilidad futura a los gobiernos locales.

Orduz, O., Pineda, J., en la revisión del concepto de gobernanza se da a conocer las bondades y los límites que el marco conceptual definido presentó para el análisis de la gobernanza de la política de infancia en Colombia. La mayoría de los autores ha coincidido en que la gobernanza surge para explicar el nuevo contexto de redefinición del papel de los gobiernos y la importancia de la acción conjunta de los diversos actores en la sociedad. Augusto, M., Bocanegra, E.,Rousseau S., involucrar a la sociedad civil organizada y con expertise en los temas de reforma, hacerla partícipe de las políticas, o incluso diseñar políticas que alineen los intereses del Estado con la sociedad, sirven como garantía de continuidad. Resaltar esta relevancia de la sociedad civil para mejorar la implementación de leyes y políticas a nivel subnacional tiene implicancias teóricas y prácticas. Delamaza, G;Thayer, L., desde el punto de vista de la gobernanza democrática, no debiera orientarse necesariamente a redefinir las regiones y buscar un marco territorial distinto para potenciar políticas públicas basadas en una articulación entre Estado y sociedad. Debiera centrarse, 
más bien, en pensar las regiones atendiendo a su heterogeneidad territorial como un ecosistema holistico. Diez, J., en la escena territorial, la idea de governance o gobernanza ha asumido la forma de las denominadas agencias de desarrollo, organizaciones de carácter público o privado donde confluyen el gobierno local y actores de la producción y el trabajo. El Coprodesu constituye una entidad de estas características que ha encarado el desafío de transformar la estructura económica de Coronel Suárez, a partir de un involucramiento activo con las problemáticas de los diferentes sectores que motorizan la actividad local.

Martínez, N., Espejel I., finalmente, es previsible que en México la investigación sobre la gobernanza ambiental continúe acrecentándose en la medida en que se analice la influencia de los procesos de interacción entre los actores sociales y gubernamentales en el sector ambiental. Por este motivo, realizar una aportación para clarificar el uso de la teoría de la gobernanza en este sector puede beneficiar a las futuras investigaciones en la materia.

Es necesario determinar una eficiente gobernanza ciudadana con un análisis territorial consensuado; no solo es utilizar los instrumentos de gestión sino fortalecer alianzas nacionales e internacionales, pensando siempre en el impacto de la gestión por procesos por resultados y estratégicos para una gobernabilidad en Estado de Derecho se debe ser un gestor eficiente de la entrega de bienes y servicios y debe ser un ente con capacidad prospectiva y respuesta frente a las oportunidades de desarrollo sostenible y sustentable de sus regiones; ya que el ente es, existe y debe seguir existiendo

\section{CONCLUSIÓN O CONSIDERACIONES FINALES}

La revisión sistemática de los 9 artículos científicos, sobre gobernanza ciudadana con análisis territorial en el Perú y en el mundo, corresponde a diferentes bases de datos, todos ellos corresponden al tipo y diseño de estudios descriptivos que concluyen en $80 \%$ que el tipo de gestión debe ser participativa por resultados en su formulación para crear desarrollo económico, político, social, tecnológico y ambiental, debido a que la gobernabilidad democrática para el Perú, implica la plena vigencia de los derechos humanos, sin discriminación, acceso a la justicia, prevención y gestión de conflictos, seguridad ciudadana, confianza en los políticos y un gobierno descentralizado, eficiente y transparente. Por otro lado el $20 \%$ concluye que la gobernanza ciudadana debe estar al alcance de aquellas personas que disfrutan un mínimo de seguridad material, educación y acceso a la información; mientras que la estrategia para el desarrollo humano es promover la participación mediante la gestión democrática de asuntos públicos donde involucre el consenso lo que invita poner mayor atención no solo en la formulación, sino también en el proceso político, reconociendo que las categorías de las políticas están atravesadas por los diversos enfoques políticos. 
Sin embargo, la gobernanza ciudadana es desdeñada en el proceso por los técnicos y, principalmente, por aquellos funcionarios que miran con recelo las instancias de control y rendición de cuenta ante sus representados. es decir, se cumple lo que farinós dasí (2008) señala para el caso español: se realizan los procesos participativos para cumplir con los requisitos formales establecidos por ley, pero sin ningún compromiso por abrir el debate verdaderamente sobre las formas de ocupación del territorio. así, los planes estratégicos con análisis territorial terminan siendo el resultado del trabajo de funcionarios y técnicos que difícilmente expresen los deseos e identidades del conjunto de la población por su interculturalidad debe ser multisectoriales con enfoque de género. Por ello, la gestión por el Ministerio de Economía y Finanzas, no solo debe ser eficiente y descentralizada sino debe ser con enfoque a la calidad del gasto según las tendencias de los planes estratégicos de desarrollo que se deben analizar en forma continua según territorio regional por lo que existe una crisis ambiental global y de inequidad social que se necesitan líderes en gestión del cambio y práctica del desarrollo sugiero formar gerentes capacees de resolver de manera innovadora la crisis aprovechando las oportunidades y proponiendo soluciones de bienestar económico con equidad social, sostenibilidad y sustentabilidad.

\section{LISTA DE REFERENCIAS}

Dalla M. (2016). Gobernanza territorial y los planes de ordenamiento territorial: Universidad Nacional de Colombia caso de la provincia de Mendoza, Argentina.v27n1.47597. consulta 12.12.16.https://doi.org/10.15446/bitácora

Orellana, A., Mena, J., Montes, V., Montes M. (2016). Plan de desarrollo comunal: ¿el instrumento rector municipal en chile? revista irvi 31(87): 173-200, agosto de 2016. Universidad Central de Venezuela. Caracas Venezuela. Consulta 02.07.18. http://www.sustentabilidades.usach.cl/sit es/sustentable/files/paginas/02-03.pdf.

Álvarez P. (2009). Actitudes ambientales y conductas sostenibles. implicaciones para la educación ambiental. revista de psicodidáctica, vol. 14, núm. 2, 2009, pp. 245-260. Madrid, España. Consulta 4.07.18 http://www.redalyc.org/pdf/175/17512724006.pdf / España

Piñeiro C, Díaz M. (2012). Perdiéndonos en la ciudad: el consumo responsable como mensaje, la ciudad de Madrid como ecosistema comunicativo. J Athenea digital. revista de pensamiento e investigación social, vol. 12, núm. 1, 2012, pp. 67-88. Barcelona España 2012. consulta 03.07.18 http://www.redalyc.org/articulo.oa?id=53723265005

Grimaldo M. (2010). Calidad de vida y estilo de vida saludable en un grupo de estudiantes de posgrado de la ciudad de lima. pensamiento psicológico, vol. 8, núm. 15, 2010, pp. 17-38. Colombia 2010. Consulta 03.07.18. http://www.redalyc.org/articulo.oa?id=80115648003

Alonso L, Fernández C, Ibáñez R. (2014). Crisis y nuevos patrones de consumo: discursos sociales acerca del consumo ecológico en el ámbito de las grandes ciudades españolas. Empiria. 
Revista de metodología de las ciencias sociales, núm. 29, septiembre-diciembre, 2014, pp.1338. consulta 03.07.18 http://revistas.uned.es/index.php/empiria/article/view/12939

Fraj E, Martínez E. (2004). El consumo ecológico explicado a través de los valores y estilos de vida. implicaciones en la estrategia medioambiental de la empresa. cuadernos de CC.EE. y EE., $\mathrm{n}^{\circ}$ 46, 2004, pp. 33-54. España 2004. consulta 03.07.18 https://dialnet.unirioja.es/servlet/articulo? codigo $=1119490$

Flores T. (2009). Comunicación para la vida saludable. hacia la promoción del desarrollo sostenible de Latinoamérica. salud, volumen 15, no.1, enero - junio la paz bolivia 2009. 2010, págs. 128 - 143 ISSN 0121-7577. http://www.bantaba.ehu.es/sociedad/files/colombia 2010. $\quad$ consulta $\quad$ view/comunicacion_para_el_desarrollo_s http://www.scielo.org.co/pdf/hpsal/v15n1 ostenible_de_latinoamerica.pdf?revision_ /v15n1a09.pdfcolombia; id=62826\&package_id=33905

Alea a. breve historia de la educación ambiental: del conservacionismo hacia el desarrollo sostenible. reino unido 2009. Consulta 04.07.18 publicado en revista futuros No. 12. http://www.revistafuturos.info

Giraldo O, Toro M, Macías A, Valencia C, Palacio S. (2010). La promoción de la salud como estrategia para el fomento de estilos de vida saludables. Hacia la Promoción de la Salud, Volumen 15, No.1, enero - junio 2010, págs. 128 - 143 ISSN 0121-7577 http://www.scielo.org.co/pdf/hpsal/v15n1/v15n1a09.pdf 